Stem-cell based gene delivery mediated by cationic niosomes for bone regeneration. Attia, N., Mashal, M., Grijalvo, S., Eritja, R., Zárate, J., Puras, G., Pedraz, J.L. Nanomedicine: Nanotechnology, Biology, and Medicine, 14(2), 521-531 (2018). PMID: 29157978, doi: 10.1016/j.nano.2017.11.005

\title{
Stem cell-based gene delivery mediated by cationic niosomes for bone regeneration
}

Noha Attia ${ }^{\mathrm{a}, \mathrm{b},{ }^{*}}$, Mohamed Mashal ${ }^{\mathrm{a},{ }^{*}}$, Santiago Grijalvo ${ }^{\mathrm{c}, \mathrm{d}}$, Ramon Eritja ${ }^{\mathrm{c}, \mathrm{d}}$, Jon Zárate ${ }^{\mathrm{a}, \mathrm{d}}$, Gustavo Puras ${ }^{\text {a,d,\# }}$ and José Luis Pedraz ${ }^{\mathrm{a}, \mathrm{d}, \# \text {. }}$

${ }^{a}$ NanoBioCel Group, Laboratory of Pharmaceutics, School of Pharmacy, University of the Basque Country (UPV/EHU), Paseo de la Universidad 7, 01006 Vitoria-Gasteiz, Spain

${ }^{\mathrm{b}}$ Histology and Cell Biology Department, Faculty of Medicine, University of

Alexandria, Alexandria, Egypt.

${ }^{c}$ Institute of Advanced Chemistry of Catalonia (IQAC-CSIC), Spain.

${ }^{\mathrm{d}}$ Biomedical Research Networking Centre in Bioengineering, Biomaterials and Nanomedicine (CIBER-BBN), Spain

*These authors have contributed equally to this work

\#Co-corresponding authors: José Luis Pedraz, and Gustavo Puras. Laboratory of Pharmacy and Pharmaceutical Technology, School of Pharmacy, University of the Basque Country, 01006, Vitoria-Gasteiz, Spain. E-mail address: joseluis.pedraz@ehu.eus and gustavo.puras@ehu.eus Phone: + (34)-945013091. Fax number: + (34)-945013040

\begin{abstract}
.
Bone morphogenetic protein-7(BMP-7) plays a pivotal role in the transformation of mesenchymal stem cells (MSCs) into bone. However, its impact is hampered due to its short half-life. Therefore, gene therapy may be an interesting approach to deliver BMP7 gene to D1-MSCs. In this manuscript we prepared and characterized niosomes based on cationic lipid 2,3-di(tetradecyloxy)propan-1-amine, combined with polysorbate 80 for gene delivery purposes. Niosomes were characterized and combined initially with pCMS-EGFP reporter plasmid, and later with pUNO1-hBMP-7 plasmid to evaluate osteogenesis differentiation. Additionally, specific blockers of most relevant endocytic pathways were used to evaluate the intracellular disposition of complexes. MSCs transfected with niosomes showed increased growth rate, enhanced alkaline phosphatase activity (ALP) and extracellular matrix deposition which suggested the formation of osteoblast-like cells. We concluded that hBMP- 7-transfected MSCs could be considered not only as an effective delivery tool of hBMP-7, but also as proliferating and bone forming cells for bone regeneration.
\end{abstract}

Key words: bone regeneration, stem cells, gene delivery, niosomes 


\section{Background}

Bone marrow-derived mesenchymal stem cells (BM-MSCs) have a multi-directional differentiation capacity into bone, cartilage, and epithelial cells when cultured in the appropriate inductive media.1 Additionally, BM-MSCs are very appealing cells due to their immunomodulatory features, allowing for allogeneic administration.2,3 When they are genetically modified, their ability to regenerate morbid osseous tissue is notably enhanced, which provides a promising ex vivo approach for clinical orthopedic applications.4,5 Several genes have been delivered and overexpressed in MSCs to promote both proliferation and differentiation into osteoblastic cells. 6

Bone morphogenetic protein-7 (BMP-7) is known to be beneficial for attachment, proliferation, as well as for differentiation of pre-osteoblasts.6,7 Although the use recombinant human BMP-7 protein, also known as osteogenic protein-1 (OP-1) was approved by FDA to improve the osteogenic differentiation of MSCs, its local stimulatory impact is hampered by diffusion and/or degradation leading to a short halflife.8 Therefore, MSC-based hBMP-7 gene therapy represents an interesting approach since it could boost bone repair by prolonged protein production in a more physiologic manner. Furthermore, MSCs supply can be crucial, especially when the number of MSCs is reduced in osteoporosis.9

Non-viral vectors have attracted great attention as safer alternatives of viral-based gene delivery vehicles since they can avoid several concerns such as; immunogenic, mutagenic and oncogenic effects.10 Additionally, non-viral gene carriers render the ability to deliver large-sized genes and are of low production costs. Consequently, the applications of non-viral vectors in clinical trials have increased considerably since 2004.11 However, non-viral formulations are still limited, mainly by their modest transfection efficiency. Therefore, this topic demands great efforts of the scientific community.12 Niosomes as self-assembled vesicular nano carriers are composed of nonionic surfactants and cationic lipids.13 Being chemically stable, easily handled and welltolerated formulations, 14 niosomes are considered advantageous over liposomes.15

Our group has recently reported encouraging in vitro and in vivo gene delivery applications of a liposomal formulation based on the novel cationic lipid 2,3di(tetradecyloxy)propan-1- amine.16 In light of such findings, our main goal was the development of a novel noisome formulation based on the aforementioned cationic lipid combined with polysorbate 80 to deliver and transfect hBMP-7 plasmid in MSCs. Additionally, the impact of such transfection on MSC in vitro osteogenic differentiation was evaluated. Firstly, niosomes were elaborated by the reverse phase evaporation technique, and characterized in terms of size, superficial charge and morphology. As a proof of concept of successful gene delivery and expression, pCMS-EGFP reporter plasmid was used to obtain nioplexes in order to transfect D1-MSCs, before the transfection efficiency evaluation of nioplexes based on pUNO1-hBMP-7 plasmid. Finally, the impact of hBMP-7 gene expression on cell osteogenic differentiation was analyzed with alkaline phosphatase activity (ALP), Von Kossa staining and ultrastructural analysis by transmission electron microscopy (TEM).

\section{Methods}

\subsection{Production of cationic niosomes}

The hydrochloride salt of the cationic lipid 2,3-di (tetradecyloxy)propan-1-amine (DTPACI) was synthesized by a slight modification of the experimental protocol described previously.17 
Once the cationic lipid was produced, niosomes were elaborated by modified reverse phase evaporation technique.18 Briefly, 5mg of cationic lipid were dissolved in $1 \mathrm{ml}$ of dichloromethane, and then emulsified in $5 \mathrm{ml}$ of polysorbate $80(\mathbf{P 8 0})(0.5 \% \mathrm{w} / \mathrm{v})$. The emulsion was obtained by sonication (Branson Sonifier 250®, Branson Ultrasonics Corporation, Danbury, USA) at $45 \mathrm{~W}$ for $30 \mathrm{~s}$. Upon dichloromethane evaporation, a dispersion containing the nanoparticles was formed by precipitation of the cationic nanoparticles in the aqueous medium. The resulting niosomes referred as DP80 contained both DTPA-Cl and P80 at a molar ration of 1:2.

\subsection{Plasmid propagation and elaboration of nioplexes}

pCMS-EGFP reporter plasmid (5541 bp, PlasmidFactory, Bielefeld, Germany), was propagated, purified and quantified as previously described.19 pUNO1-hBMP-7 plasmid $(4497 \mathrm{bp})$ was purchased from InvivoGen (Toulouse, France). Nioplexes (niosome/DNA complexes) were elaborated by mixing an appropriate volume of a stock solution of pCMS-EGFP/pUNO1- hBMP-7 plasmids $(0.5 \mathrm{mg} /)$ with different volumes of niosome suspensions ( $1 \mathrm{mg}$ cationic lipid/ml) to obtain different cationic lipid/DNA ratios $(\mathrm{w} / \mathrm{w})$. The mixture was left for $30 \mathrm{~min}$ at room temperature to enhance electrostatic interaction. Nioplexes were referred as DP80-EFGP or DP80-hBMP-7 depending on the plasmid used.

\subsection{Characterization of niosomes and DP80-EFGP nioplexes}

Particle size and zeta potential (ZP) were determined by dynamic light scattering (DLS) and Laser Doppler Velocimetry (LDV) (Zetasizer Nano ZS, Malvern Instruments, UK). Particle size was obtained by cumulative analysis. All measurements were carried out in triplicate. The morphology of nioplexes was assessed by cryo-TEM analysis.20 Digital images were acquired for samples examined by TEM, TECNAI G2 20 TWIN (FEI), operating at an accelerating voltage of $200 \mathrm{KeV}$ in a bright-field and low-dose image mode. The capacity of niosomes to condense, release and protect DNA from enzymatic digestion was performed by agarose gel electrophoresis assay using pCMS-EGFP plasmid. Naked DNA (as control) or niosome-complexed DNA samples (200 ng of plasmid/20 $\mu \mathrm{l})$ were run on agarose gel $(0.8 \% \mathrm{w} / \mathrm{v})$, stained with GelRed ${ }^{\mathrm{TM}}$. The gel was immersed in a Tris-acetate-EDTA buffer and exposed for $30 \mathrm{~min}$ to $120 \mathrm{~V}$. Bands were visualized by ChemiDoc ${ }^{\mathrm{TM}}$ MP Imaging System (Bio-Rad, Madrid, Spain). To analyze DNA release from nioplexes at different cationic lipid/DNA mass ratios, $20 \mu 1$ of a $2 \%$ SDS solution (Sigma-Aldrich, Madrid, Spain) was added to each sample. Protection capacity of nioplexes against enzymatic digestion was studied after adding DNase I (Sigma-Aldrich, Madrid, Spain) at a final concentration of $1 \mathrm{U}$ DNase I/2.5 $\mu$ g DNA. Afterwards, mixtures were incubated at $37^{\circ} \mathrm{C}$ for $30 \mathrm{~min}$. Finally, a $2 \%$ SDS solution was added to release DNA from nioplexes.

\subsection{Culture and characterization of D1-MSCs}

Mouse mesenchymal stem cells (ATCC ${ }_{\circledast}$ CRL-12424 тм) (D1-MSCs) were cultured in growth medium (GM) composed of: Dulbecco's modified Eagle's medium (DMEM) (ATCC 30-2002) supplemented with 10\% fetal bovine serum (FBS) and 1\% penicillin/streptomycin, (both reagents from Gibco, Spain). Cell multipotency was determined by differentiation into osteogenic and adipogenic lineages as reported in previous work. 1 After two weeks, cells were stained with Alizarin Red S (osteogenic differentiation) and Oil Red O (adipogenic differentiation).

\subsection{Reporter EGFP plasmid in vitro transfection}


D1-MSCs were seeded at a density of $9 \times 10_{4}$ cells/well in 24-well plates. $24 \mathrm{~h}$ later, GM was removed and cells were washed with serum-free Opti-MEM® solution (Gibco®, California, USA). Then, cells were exposed to DP80-EFGP nioplexes (1.25 $\mu \mathrm{g}$ DNA) at different cationic lipid/DNA ratios (w/w), and incubated in serum-free Opti-MEM ${ }_{\circledast}$ solution for $4 \mathrm{~h}$ at $37^{\circ} \mathrm{C}$. Subsequently, transfection medium was replaced with GM, and cells were allowed to grow for further $48 \mathrm{~h}$ until fluorescence microscopy imaging (Nikon TSM) and FACSCalibur flow cytometer analysis (BD Biosciences, USA). To analyze cell viability, cells were stained with Propidium Iodide (Sigma-Aldrich, Madrid, Spain) prior to flow cytometer analysis. Experiments with uncomplexed DNA was considered as negative control. Regarding to positive control, both TransIT ${ }^{8} 2020$ (Mirus, Madrid, Spain) (at a ratio of $3 \mu$ l of reagent/1 $\mu \mathrm{g}$ of DNA) and Lipofectamine ${ }^{\circ}$ 2000 (L2000) (Invitrogen, California, USA) were used. A minimum of 10.000 events were acquired and analyzed for each sample in triplicate.

\subsection{Endocytosis uptake mechanism for nioplexes}

Several uptake inhibitors were used to determine the endocytosis mechanism by nioplexes. Genistein and chlorpromazine hydrochloride were used as inhibitors for caveolae-mediated endocytosis (CvME) and clathrin-mediated endocytosis (CME), respectively. Methyl- $\beta$ - cyclodextrin was used to simultaneously inhibit $\mathrm{CvME}$ and CME. Additionally, wortmannin, was employed to inhibit macropinocytosis (MPC).

D1-MSCs were transfected as mentioned in the previous 2.5 section, but in this case, nioplexes were prepared with FITC-labeled EGFP plasmid (DareBio, Madrid, Spain). Cells were incubated for $30 \mathrm{~min}$ with genistein $200 \mu \mathrm{M}$, methyl- $\beta$-cyclodextrin $5 \mathrm{mM}$ and wortmannin $50 \mathrm{nM}$, and for $60 \mathrm{~min}$ with chlorpromazine hydrochloride $5 \mu \mathrm{g} / \mathrm{ml}$ prior to addition of nioplexes. After $4 \mathrm{~h}$ of incubation with nioplexes, cells were washed thoroughly with PBS, detached, and analyzed by FACSCalibur flow cytometer (BD Biosciences, USA). For each sample, 10.000 events were collected and analyzed. Cellular uptake data were expressed as the percentage of FITC-positive cells. Naked DNA was used as a negative control. Each sample was analyzed in triplicate.

\subsection{Characterization of DP80-hBMP-7 nioplexes, in vitro transfection and proliferation/cytotoxicity assay.}

DP80-hBMP-7 nioplexes were characterized at different mass ratios as previously described in the section 2.3. Subsequently, transfection of D1-MSCs was performed using DP80-hBMP-7 nioplexes using the transfection protocol described in section 2.5. After $48 \mathrm{~h}$ post-transfection, conditioned media was collected, filtered ( $0.22 \mu \mathrm{m}$ filters) and preserved at $-80^{\circ} \mathrm{C}$ until ELISA was performed (R\&D, UK) to determine the hBMP-7 secreted. CCK-8 viability/proliferation assay (Sigma Aldrich, Spain) was performed according to manufacturer's instructions. Color development was read at 450 $\mathrm{nm}$ (Tecan M200 microplate reader), corrected with reference wavelength at $690 \mathrm{~nm}$ and normalized against mean value of three blank wells (GM).

\subsection{In vitro assessment of osteogenesis}

Transfection protocol of DP80-hBMP-7 nioplexes was applied as mentioned in section 2.5. On day 2 post-transfection, both cell cultures in transfected and untransfected (control) wells were allowed to continue in growth medium/ osteogenic medium (GM/OM) to determine the impact of hBMP-7 transfection on the osteogenesis potential of D1-MSCs. Therefore, cells were classified in four different groups; untransfected 
culture in GM/OM, transfected culture in GM/OM. After 2 weeks, the following in vitro assays were performed.

\subsubsection{Alkaline Phosphatase (ALP) activity assay}

D1-MSCs from the four groups were washed and $500 \mu \mathrm{l}$ of GM were added. After $24 \mathrm{~h}$ of incubation, conditioned media were collected, filtered $(0.22 \mu \mathrm{m}$ filters $)$ and ALP activity assay was performed according to manufacturer's instructions. ALP activity of each sample was read at $405 \mathrm{~nm}$ (Tecan M200 microplate reader) and normalized to 100.000 cells.

\subsubsection{Von Kossa Staining}

For qualitative assessment of calcium deposits, Von Kossa staining (Abcam, UK) was performed as previously reported.21 Micrographs were digitally processed using NIH Fijiø program.

\subsubsection{Transmission electron microscopy (TEM)}

D1-MSCs from both untransfected and transfected groups cultured in GM for two weeks were detached and pellets were fixed with $2 \%$ gluteraldehyde $(\mathrm{pH} \mathrm{7.4)}$ for $24 \mathrm{~h}$ at $4^{\circ} \mathrm{C}$, processed as previously reported 1 and examined with Philips EM208S TEM.

\subsection{Statistical analysis}

Statistical differences between groups at significance levels of $>95 \%$ were calculated by ANOVA and Student's $t$ test. In all cases, $P$ values $<0.05$ were regarded as significant. Normal distribution of samples was assessed by the Kolmogorov- Smirnov test and the homogeneity of the variance by the Levene test. Numerical data were presented as mean $\pm \mathrm{SD}$.

\section{Results}

\subsection{Physicochemical characterization of niosomes and DP80-EGFP nioplexes}

The size of niosomes was $54.0 \pm 0.9 \mathrm{~nm}$ with PDI vales of $0.5 \pm 0.1$ and $\mathrm{ZP}$ values of $41.9 \pm 7.1 \mathrm{mV}$. Figure 2-A depicts size and $\mathrm{ZP}$ values of nioplexes at mass ratios from $2 / 1$ to $20 / 1$. The size of nioplexes (bars) decreased from $168 \mathrm{~nm}$ to $84 \mathrm{~nm}$ at cationic lipid/DNA mass ratios of $2 / 1$ and 16/1, respectively, then increased slightly to $111 \mathrm{~nm}$ at 20/1 mass ratio. PDI of all nioplexes were less than 0.3 (Table 1, supplementary data). Concerning ZP, readings were clearly increasing from $-28.9 \mathrm{mV}$ at $2 / 1$ mass ratio to $+19.2 \mathrm{mV}$ at $8 / 1$ mass ratio, while afterwards depicted no notable change. As illustrated in Fig. 2-B, the cryo-TEM-assessed nioplexes (16/1 mass ratio) exhibited a discrete imperfectly spherical morphology with no aggregates. Some nioplexes depicted lamellar pattern at higher magnification (arrows, Fig 2-B inset). More pictures at lower magnification can be observed in Figure 1, on supplementary data. Figure 2-C represents gel retardation assay of DP80-EGFP nioplexes prepared at different cationic lipid/ DNA ratios (8/1, 12/1, 16/1 and 20/1). Partial condensation of DNA was seen since faint SC bands were observed on 4, 7, 10 and 13 lanes. However, condensed DNA was released upon the addition of SDS, as can be observed on lanes 5, 8, 11 and 14 . Moreover, complexed DNA was protected against DNAse I enzymatic digestion, since clear OC (open circular) and SC (supercoiled) bands were detected on lanes 6, 9, 12 and 15 compared to the absence of OC/SC bands in lane 3 (free DNA).

\subsection{In vitro culture, transfection and viability of MSCs by reporter EGFP plasmid}


Cultured D1-MSCs were spindle-shaped adherent, colony forming cells (Fig 3-A1). Upon culture in osteogenic or adipogenic media, they demonstrated calcific deposits by Alizarin Red S (Fig 3-A2) and Oil red O-stained cytoplasmic lipid vacuoles (Fig 3-A3), respectively. In figure 3-B, all cationic lipid/DNA ratios (w/w) studied above 4/1 showed considerable percentages of transfection. Meanwhile, naked DNA (control) did not elicit any transfection. The mass ratio of $16 / 1$ represented the best transfection results of $35.9 \pm 1.9 \%$, which did neither differ significantly from that obtained by TransIT 2020® $(40.7 \pm 2.5 \%)$ nor by L2000 $(35.06 \pm 0.5 \%)$. On the other hand, cell viability was higher with TransIT 2020® $(\mathrm{p}<0.05)$ compared to mass ratio of 16/1 $(99.1 \pm 0.5 \%$ and $93.6 \pm 1.2 \%$, respectively). Regarding mean fluorescence intensity (MFI) obtained by our nioplexes at $16 / 1$ as mass ratio, it was markedly higher than that obtained by TransIT 2020® (1792 a.u. and 1006 a.u, respectively). Similar finding was

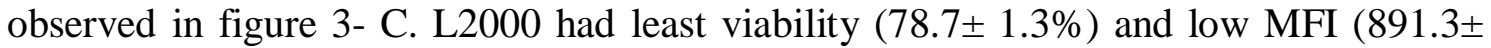
2.7 a.u.), therefore excluded from further experiments.

\subsection{Endocytosis uptake mechanism of nioplexes}

The uptake of nioplexes at 16/1 mass ratio was determined compared to naked DNA (negative control). Figure 4-A showed representative dot plots for uptake of both free DNA and DP80 nioplexes ( $0.32 \%$ and $83 \%$, respectively).

Interestingly, we observed that the selective inhibition of caveolae-mediated (genistein) or clathrin-mediated (chlorpromazine hydrochloride) uptake pathway had significant effects on cellular uptake of nioplexes $(p<0.05)$. However, there was no statistically significant difference between both inhibitors. When both previously mentioned endocytosis mechanisms were simultaneously inhibited by prior treatment with methyl$\beta$-cyclodextrin, an extremely significant difference was observed when compared to control group $(\mathrm{p}<0.0001)$. Nevertheless, wortmannin, an inhibitor of MPC, did not affect the nioplexes internalization $(\mathrm{p}>0.05)$.

\subsection{Physicochemical characterization of DP80-hBMP-7-nioplexes}

Figure 5-A depicts size and ZP values of nioplexes at different mass ratios (from 2/1 to 20/1). The size of nioplexes (bars) decreased gradually from $217 \mathrm{~nm}$ at $2 / 1$ cationic lipid/DNA mass ratio to $69 \mathrm{~nm}$ at 20/1 mass ratio with PDI values less than 0.3 (Table 1, supplementary data). Regarding ZP values, readings were clearly increasing from $11.1 \mathrm{mV}$ at $2 / 1$ mass ratio to $+26.2 \mathrm{mV}$ at $8 / 1$ mass ratio, while afterwards, slowly decreased to $+18.3 \mathrm{mV}$ at mass ratio of $20 / 1$. As illustrated in figure 5-B, nioplexes, assessed by cryo-TEM, exhibited a discrete spherical/ovoid morphology with no notable clumps. Some nioplexes appeared lamellar at higher magnification (arrows, Fig. 5-B inset). More pictures at lower magnification can be observed in Figure 1, on supplementary data. Figure 5-C illustrated that DNA was partially condensed at mass ratio of 4/1, though complete capture was discerned at mass ratios of 8/1, 12/1, and 16/1, since no SC bands were observed on lanes 7, 10 and 13. Complexed DNA was released after the addition of SDS, as it could be observed on lanes 5, 8, 11 and 14. Except for mass ratio of 4/1, captured DNA was protected against DNAse I enzymatic digestion since clear $\mathrm{OC} / \mathrm{SC}$ bands were detected on lanes 9, 12 and 15 in comparison to lane 3 (free DNA) due to the unopposed lytic action of the enzyme.

\subsection{Transfection of MSCs by pUNO1-hBMP-7 plasmid}

As can be observed in figure 6 (bars), all studied cationic lipid/DNA ratios (w/w) demonstrated significant secretion of hBMP-7 protein in comparison to the untransfected cells (controls). The mass ratios of 4/1, 8/1 and 12/1 demonstrated the 
best transfection results $(1460 \mathrm{pg} / \mathrm{ml})$, without significant differences observed among them ( $\mathrm{p}>0.05)$, but was still less than secretion obtained by TransIT 2020® transfection $(2111 \mathrm{pg} / \mathrm{ml})(\mathrm{p}<0.05)$. On the other hand, cell counting assay (CCK8) performed immediately after conditioned media was collected ( $48 \mathrm{~h}$ post-transfection) demonstrated that transfection by nioplexes at ratios of 8/1,12/1, and 16/1 induced significant cell proliferation $(164 \%, 137 \%$ and $122 \%$, respectively) compared to control and TransIT $2020 \circledast$ groups (100\% and 23\%, respectively) (Fig 6, line). Nevertheless, cell viability decreased significantly to $65 \%$ at $20 / 1$ cationic lipid/DNA mass ratio.

\subsection{In vitro assessment of osteogenesis}

\subsubsection{Alkaline Phosphatase (ALP) activity assay}

As observed in figure 7-A, ALP activity in transfected cell culture was significantly higher whether cells were cultured in GM or OM. Although ALP activity of all groups was superior to untransfected cells cultured in GM $(\mathrm{p}<0.05)$, no significant differences were discerned among those groups $(\mathrm{p}>0.05)$.

\subsubsection{Von Kossa Staining}

After Von Kossa staining, no calcium deposits were observed in the control group (Fig 7- B1). However, mineralized bone nodules were observed in D1-MSCs cultured in OM (Fig 7-B2). Similar nodules were noticed in culture plates of D1-MSCs transfected with hBMP-7, whether cultured in GM or OM (Figs 7-B3 and 7-B4, respectively).

\subsubsection{Transmission electron microscopy (TEM)}

TEM was applied to detect the ultrastructure of untransfected (7-C1) vs. transfected (7C2) D1-MSCs cultured for two weeks in GM. Electron micrographs of both groups showed large euchromatic nuclei $(\mathrm{N})$ with prominent nucleoli $(\mathrm{n})$. The chromatin was dispersed except for a thin dense layer located immediately inside the nuclear envelope. Although cytoplasmic organelles depicted no obvious change, Golgi complex (arrow head) was evident in many cells of transfected group (7-C2). In addition, deposition of amorphous extracellular matrix was noticed (arrows).

\section{Discussion}

Our novel niosomes were formulated with cationic lipid salt (DTPA-HCl) and the nonionic surfactant polysorbate 80 (Fig 1). The cationic lipid, DTPA, has been used for gene delivery applications, 22 though it was not tested neither in salt form, nor as a noisome formulation. Structurally, DTPA-HCl is composed of the four crucial elements that govern the process of gene transfection. Namely, the polar head, backbone, linker and two non-polar tails.23 During niosome elaboration, we endeavored to circumvent the potential cytotoxicity of the ether linker of DTPA-HCl by decreasing its molar ratio compared to P80 (DTPA-Cl to P80 as 1:2, respectively).

Once synthesized, DP80 niosomes showed appropriate nano-scaled size for gene delivery purposes. High positive $\mathrm{ZP}$ values $(>+25 \mathrm{mV})$ would ensure long-lasting stability24 and spontaneous electrostatic interaction with plasmid DNA. Additionally, it could enhance proper binding of the resulting nioplexes to the negatively charged cell coat prior to nioplexes internalization.23

To probe the behavior of our niosomes we elaborated, as a proof of concept, DP80EGFPnioplexes by adding pCMS-EGFP reporter plasmid to DP80 niosomes at different cationic lipid/DNA mass ratios. Such sequence was adopted to ensure better complex assembly.25 A gradual decrease in the size of nioplexes was observed (168 - $84 \mathrm{~nm}$, 
Fig.2-A), at mass ratios studied up to 16/1. Such decline in size is governed by balanced events in a delicate multistep self-assembly process of complex formation, such as: electrostatic interaction, further membrane merging, lipid mixing and aggregate growth. 25 The size of nioplexes is of great importance to grant proper interactions with cell components (organelle membranes and cytoskeletal filaments). The small size of nioplexes might be advantageous to improve the rate of cellular uptake.26 In addition, nioplexes would be able to navigate the mammalian cytoskeleton with mesh size about $200 \mathrm{~nm} .27$ Regarding the ZP values, the gradual initial increase of superficial charge along with cationic lipid/DNA ratios (w/w), followed by a plateau phase, demonstrated the ability of cationic niosomes to bind to and neutralize the negatively charged phosphate groups in plasmid DNA.28 At small cationic lipid/DNA mass ratios (2/1 and $4 / 1$ ), the ZP readings were negative and shifted to the positive territory (around +20 $\mathrm{mV}$ ) at 8/1,12/1, 16/1, and 20/1 mass ratios. At higher ratios, nioplexes were figured to function as gene delivery carriers taking advantage of their positive surface charge that facilitates proper electrostatic interaction with the anionic glycocalyx during the early steps of the endocytosis.29

The shape of complexes has a marked effect on their performance as a gene delivery candidate, 30 albeit, the effect of particle shape on biological interactions is not yet understood. As reviewed by Champion et al,30 vast observations proved the complexity of cell-particle interactions and revealed the ability of cells to respond differently to varied particle shapes. For instance, nonspherical particles were reported to be internalized faster than the perfectly spherical ones.26, 31 cryo-TEM micrographs illustrated that most of the DP80-EGFP complexes were not perfectly spherical (Fig 2B). Additionally, the discerned discrete morphology of nioplexes with no aggregates (Fig 2-B) could be attributed to the moderately positive surface charge. Interestingly, the lamellar pattern observed in several nioplexes is believed to occur during the process of complex formation by the complete topological transformation of both lipid and DNA into compact quasispherical complex particles with diameter around $200 \mathrm{~nm}$, that form string-like colloidal aggregates, inside of which, the complexes have an ordered multilamellar planar structure (Fig 2-B, arrows).32 The regular lamellar spacing of almost 5.5-6 nm (Fig 2-B inset) was similar to that reported by Le Bihan and colleagues, denoting that the repetitive motif corresponded to DNA strands complexed with cationic lipid bilayers. 33

Among other factors that can influence on the transfection process, the electrostatic interactions between the negatively charged phosphate groups of DNA and the positively charged amine groups of the cationic niosomes merits special attention.20, 28, 34, 35 We observed by agarose gel electrophoresis assay that at all cationic lipid/DNA ratios evaluated, niosomes were able to capture, release and protect the DNA from enzymatic digestion (Fig 2-C).

Once we evaluated that DP80-EGFP nioplexes would be suitable for gene delivery purposes, we proceeded to evaluate their biological performance in vitro. BM-MSCs are of vital importance in adult bone repair as they home to the fracture site, proliferate and serve as a source of osteochondral progenitors. Hence, bone marrow-derived D1-MSCs were selected for the current study. In accordance to our results (Fig 3-A) they were known to represent a multipotent MSC platform holding potential of osteogenic fate determination in vitro.36, 37 Moreover, their phenotypic characteristics and osteogenic differentiation potential were reported to be consistent with primary human MSCs.36, 38 There is a general belief that niosomes are well tolerated both in vitro and in vivo conditions.19 Our results (Fig 3-B) illustrated that DP80 (16/1) induced transfection efficiency similar to that obtained by TransIT $₫ 2020$ and L2000 commercial reagents 
(35.8\%, 40.6\% and 35.1\%, respectively). However, the MFI of the transfected cells (Figs 3-B and 3-C) were significantly higher with DP80 (1791 a.u.) compared to TransIT® 2020 (1006 a.u). Interestingly, cell viability reported with DP80-EGFP nioplexes was significantly higher than that with L2000, while inferior to that reported by TransIT $₫ 2020$ commercial reagents. Such cytotoxicity might be induced, even in part, by GFP-induced apoptosis that might result from GFP aggregations or free radicals.39 In general, the main aim of gene delivery is to attain overexpression of the transfected gene to express the protein in super physiological doses. Thereafter, transfection efficiency is not only a matter of how many cells were transfected, but also the amount of gene expression by the transfected cells.

The high transfection efficiency values observed were preceded by a great percentage of nioplexes uptake as shown for best transfection efficiency (16/1) (Fig 4-A). Despite the reported high uptake percentage (almost 83\%), the performance of non-viral vectors is known to be clearly affected by their distinct cellular internalization pathway, taking into account the variable effectiveness of every pathway in the release of DNA into the cytoplasm, which is one of the critical steps in the eventual transgene expression.19

Therefore, we further analyzed intracellular trafficking of our nioplexes by blocking three of the most important endocytosis pathways in mammalian cells, 26,10 namely, caveolae-mediated endocytosis (CvME), clathrin-mediated endocytosis (CME) and macropinocytosis (MPC).

The results observed in figure 4-B suggested that internalization of DP80-EGFP was mainly through CvME and CME with no significant difference between them. Although a robust consensus does not exist, it is widely accepted that the endolysosomal fate is the hallmark feature of CME.40, 41 Oppositely, CvME and/or MPC routes could be advantageous to avoid lysosomal degradation, which could ensure a better plasmid delivery and integrity.10,42 Consequently, the relevant contribution of the clathrinmediated endocytosis pathway, which directs nioplexes to the lysosomes, could explain the relatively low transfection efficiency values observed $(35.8 \%$, Fig 3-B), despite the fact that high number of cells (83\%, Fig 4-A) captured the complexes.

The promising results obtained with the reporter EGFP plasmid encouraged us to explore hBMP-7 gene transfection.

As done before with DP80-EGFP nioplexes, we characterized DP80-hBMP7 nioplexes regarding their physicochemical features. As noted in figure 5-A, gradual decline of size correlated to the increased cationic lipid/DNA mass ratio (bars). Starting from mass ratio of $4 / 1, \mathrm{ZP}$ values observed were in the positive territory that fluctuated between 18.3 and $26.2 \mathrm{mV}$. Particles were of no perfect shape and mostly acquired the planar lamellar pattern (Fig 5-B). Similar to what has been reported in figure 2-C, DP80hBMP-7 nioplexes were able to bind, release and protect plasmid DNA against DNase I enzymatic digestion (Fig 5-C). These findings suggested that DP80-hBMP-7nioplexes could transfect D1-MSCs in vitro. The hBMP7 protein secreted after $48 \mathrm{~h}$ of transfection (Fig 6) reached its highest value at the ratios of 4-12/1(up to $1460 \mathrm{pg} / \mathrm{ml}$ ). These results showed that the mass ratios of best transfection efficiency changed with different plasmids used (EGFP at 16/1).

The CCK-8 assay interestingly depicted that cells seemed to proliferate more significantly when transfection was the best at mass ratios of $8 / 1$ and $12 / 1$. This phenomenon might be due to two main factors; first, the negligible cytotoxicity effect of our nioplexes on D1-MSCs at such ratios compared to the higher ratios (16/1 and 20/1), and second, a direct enhancement of mitotic activity of such cells by the released hBMP-7 protein 43 . 
Although TransIT $₫ 2020$ induced significantly efficient transfection $(2111.5 \mathrm{pg} / \mathrm{ml}$, Fig. $6)$, it extremely hampered cell viability (23\%). This might be referred to the cytotoxic impact of the hBMP7- based TransIT $₫ 2020$ complexes that seemed to badly attenuate the initial population of hBMP-7secreting cells.46 Since ELISA assay was performed as early as $48 \mathrm{~h}$ posttransfection, the number of cells in TransIT® 2020 samples was not obviously enhanced by the putative mitogenic effect of hBMP-7 protein.

Noteworthy that results (shown in figs. 3-B and 6) elucidated the fact that different plasmids could behave differently in regard to transfection efficiency or cell viability. In the current study, both EGFP and hBMP-7 plasmids had a different length (5541 and $4497 \mathrm{bp}$, respectively) and promotors. Therefore, the obtained nioplexes rendered different physicochemical characteristics and thus demonstrated discrepant behavior regarding to transfection efficiency or cell tolerance. 44,45

We further assessed the effectiveness of hBMP-7 overexpression to induce osteogenesis in vitro using the alkaline phosphatase activity (Fig 7-A). ALP is considered an important parameter to assess osteoblastic phenotype as it is required for mineralization, thus induced early during osteoblast differentiation.46 Both ALP assay and Von Kossa staining (Fig 7-B) of the mineralized bone nodules denoted that hBMP-7 expression induced spontaneous osteoblastic differentiation and calcified matrix deposition under standard culture in GM. The amorphous extracellular matrix deposition was further shown at the ultrastructural level in figure 7-C2. During this process, secreted hBMP-7 was anticipated to interact with cell membrane receptors, which could induce a signaling cascade that lead to osteoblastic differentiation in autocrine and/or paracrine mode. 47 Nevertheless, BMP-7 transfection did not manifest significant difference when cells were cultured in OM for two weeks (Fig 7-B3 vs. 7-B4).

Our results suggest that hBMP-7-transfected D1-MSCs could be considered not only as an effective delivery tool of hBMP-7, but also as proliferating and bone forming cells for bone regeneration purpose, which offers reasonable hope to use stem cell-based gene delivery by niosomes approach to target sites where bone regeneration is needed. To validate that hypothesis, additional in vivo experiments and an optimization process of the niosomes formulations would be required, since biological conditions are different and not always exists a consistent correlation between in vitro and in vivo experiments.

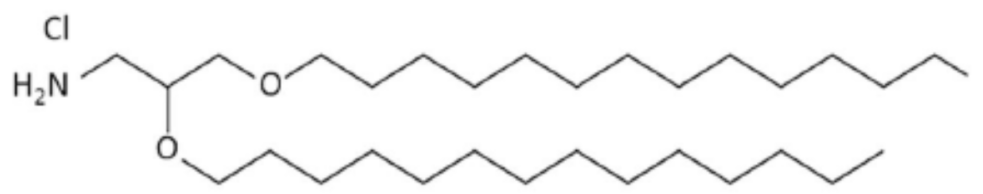

A. 2,3-Di (tetradecyloxy)propan-1-amine (DTPA Chloride salt)

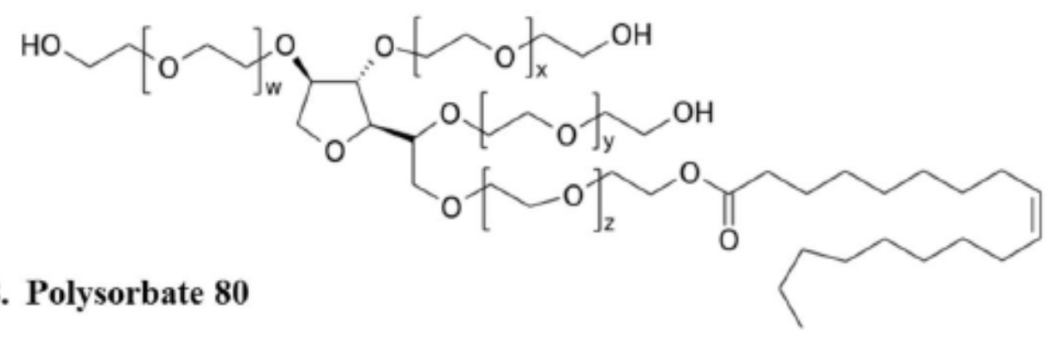

Figure 1: Chemical structure of the components of DP80 niosomes (A. Cationic lipid DTPA-Cl, B. Polysorbate 80). 

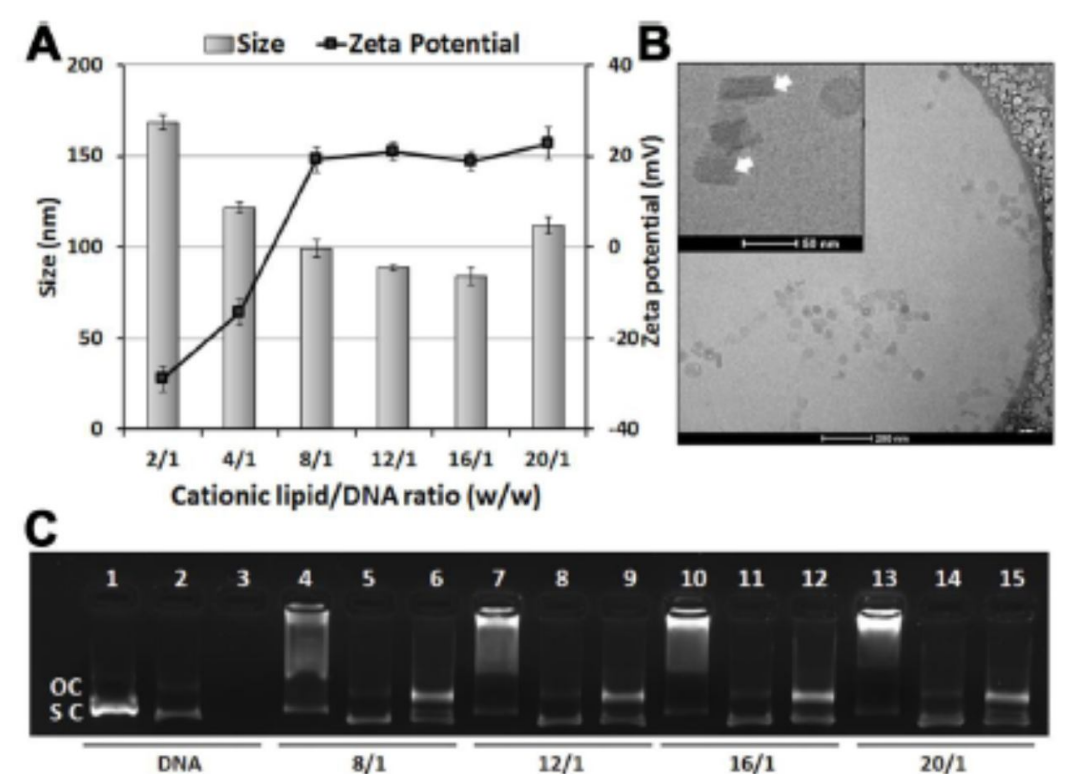

Figure 2: Physicochemical characterization of DP80-EGFP nioplexes (A) Effect of cationic lipid/DNA ratio (w/w) on both particle size (bars) and ZP (line), $n=3$. (B) Cryo-TEM micrographs of nioplexes at 16/1 cationic lipid/DNA ratio (w/w). Arrows indicate lamellar pattern. Scale bar $=200 \mathrm{~nm}$ (inset scale bar $=50 \mathrm{~nm}$ ). (C) Binding, SDS-induced release and protection of DNA at different cationic lipid/DNA ratios (w/w) of DP80-EGFP- nioplexes visualized by agarose electrophoresis. Nioplexes were treated with SDS (lanes 2, 5, 8, 11 and 14) and DNase I + SDS (lanes 3, 6, 9, 12 and 15). OC: open circular form, SC: supercoiled form.
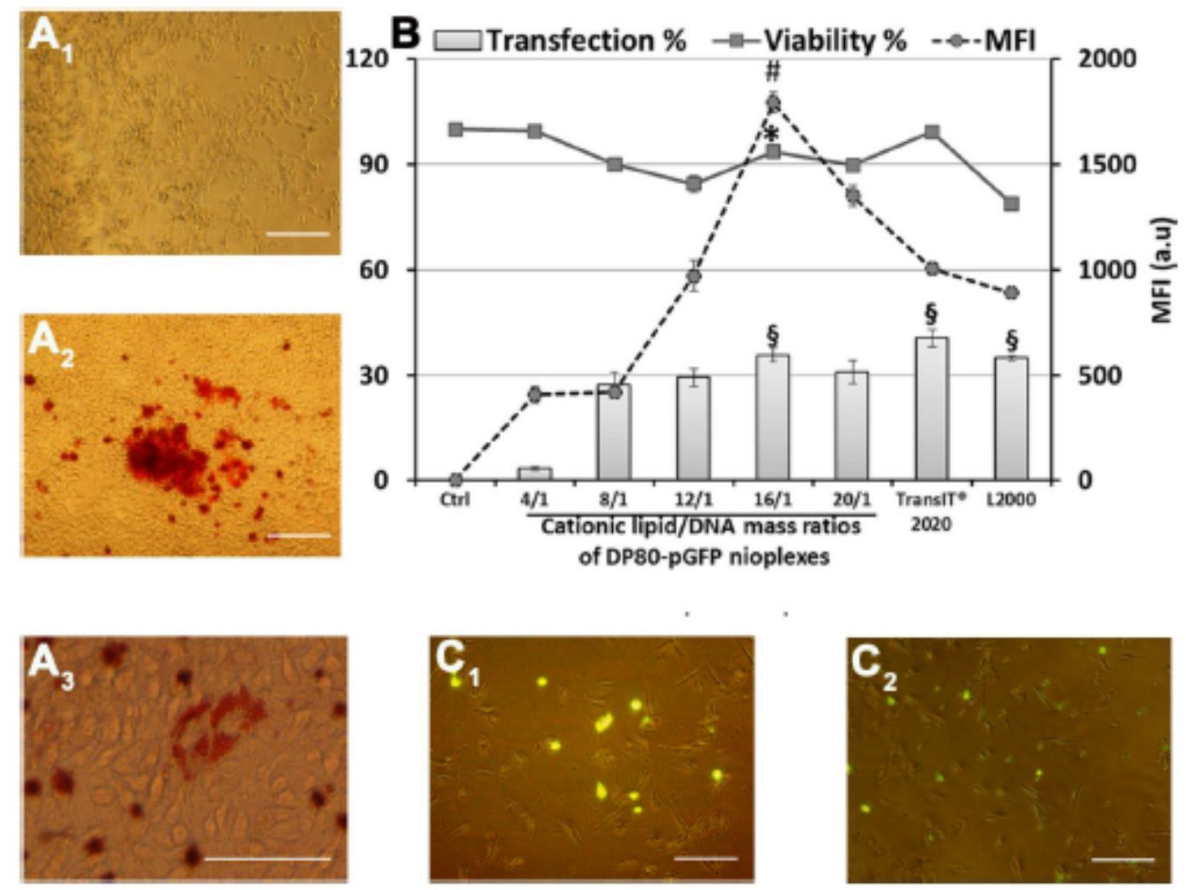
Figure 3: D1-MSCs culture (A1), osteogenic (A2) and adipogenic differentiation (A3). (B) Cell transfection efficiency and viability at different cationic lipid/DNA ratios (w/w). Percentage of EGFP-positive cells (bars), percentage of viable cells (line) and MFI (dashed line), $(\mathrm{n}=3)$. Similar symbol $(\S)$ indicate insignificant differences among three bars $(\mathrm{P}>0.05)$. $* \mathrm{P}<0.05$ vs. TransIT $\circledast 2020$ and L2000 viability. \#P $<0.05$ vs. TtansIT $₫ 2020$ and L200 MFI. (C) Representative overlay micrographs of D1-MSCs 48 h post-transfection via (C1) DP80-EGFP nioplexes (16/1 cationic lipid/DNA mass ratio) and (C2) TtansIT $\circledast 2020$. Scale bars: A1, A2, B1 and B2 $=50 \mu \mathrm{m}$ and A3 $=25 \mu \mathrm{m}$.
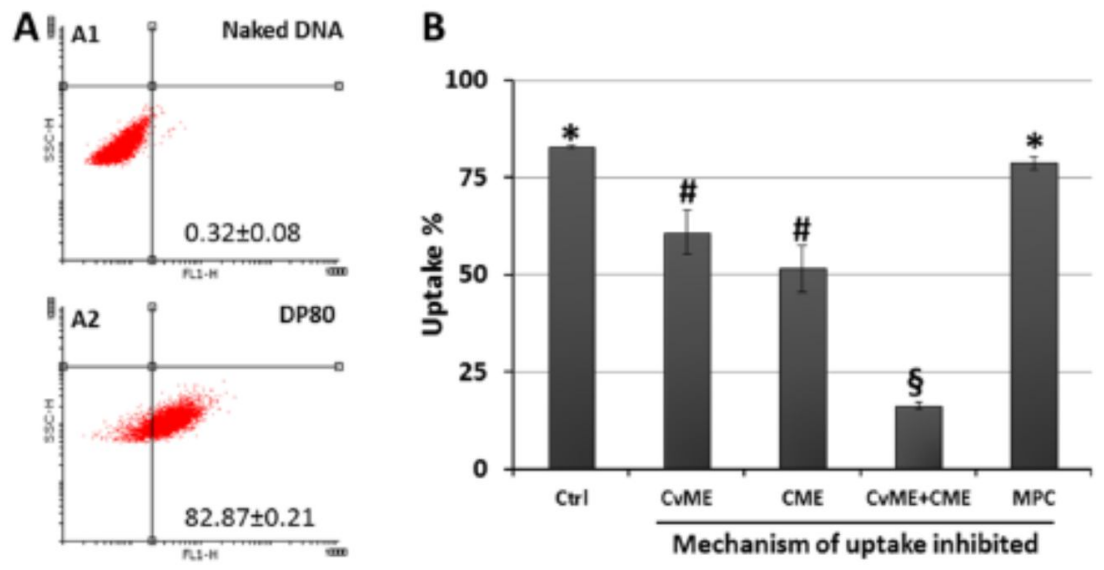

Figure 4: Uptake study of D1-MSCs 4 h post-incubation with FITC-labeled nioplexes. (A) Representative dot plots for cellular uptake, (A1) naked DNA (no carrier), (A2) DP80 nioplexes. (B) Uptake \% (FITC-positive cells) in the presence/absence of endocytosis inhibitors. (Ctrl) represents the uptake with no inhibitors. Mean $\pm \mathrm{SD} ; \mathrm{n}=$ 3. $(* \mathrm{p}<0.05, * * * \mathrm{p}<0.0001)$
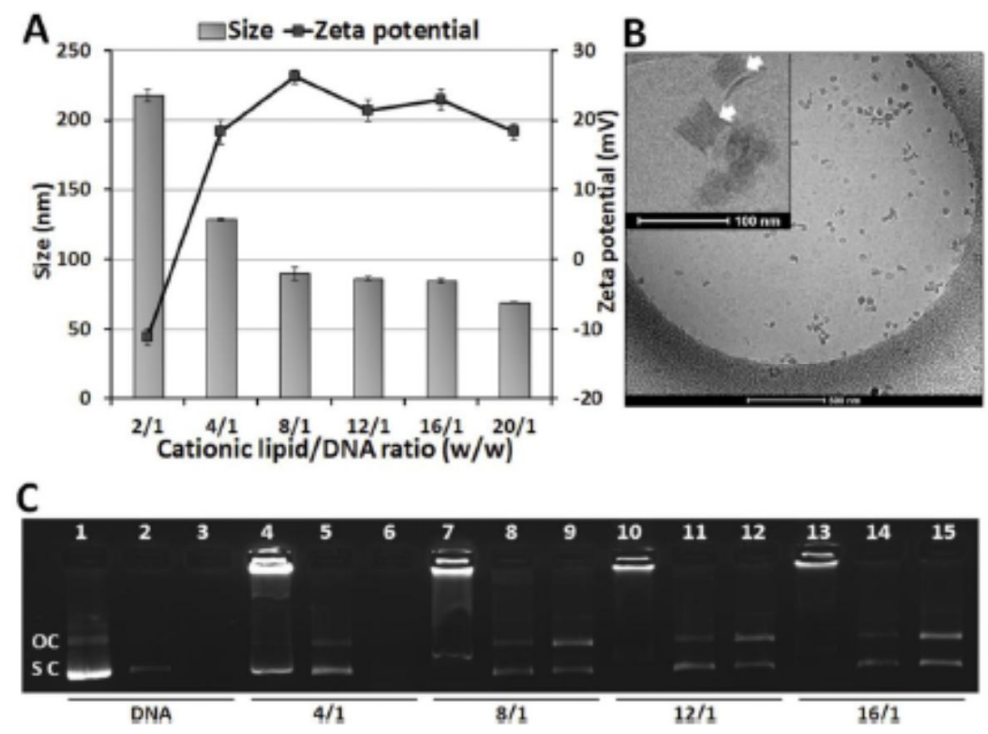

Figure 5: Physicochemical characterization of DP80-hBMP-7 nioplexes. (A) Effect of cationic lipid/DNA ratio (w/w) on particle size (bars) and zeta potential (line), $n=3$. (B) Cryo-TEM micrographs of nioplexes at ratio of $8 / 1$ cationic lipid/DNA ratio $(\mathrm{w} / \mathrm{w})$. 
Arrows indicate lamellar pattern. Scale bar $=500 \mathrm{~nm}$ (inset scale bar $=100 \mathrm{~nm}$ ). (C) Binding, SDS-induced release and protection of DNA at different cationic lipid/DNA ratios (w/w) of DP80-hBMP-7 nioplexes visualized by agarose electrophoresis. Nioplexes were treated with SDS (lanes 2, 5, 8, 11 and 14) and DNase I + SDS (lanes 3, 6, 9, 12 and 15). OC: open circular form, SC: supercoiled form.

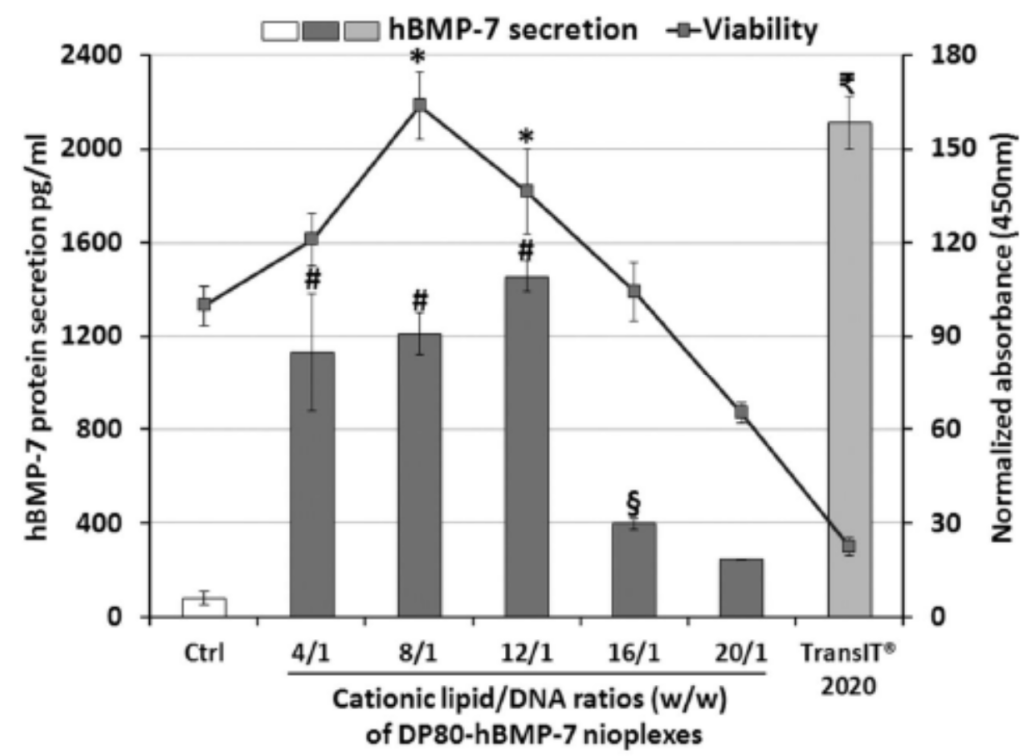

Figure 6: hBMP-7 secretion $(\mathrm{pg} / \mathrm{mL})$ compared to untransfected cells (Ctrl) and TransIT ${ }_{\circledast}$ 2020- transfected cells (Bars). CCK-8 viability assay (line). Values represent mean \pm SD $(n=3)$. Different symbols above bars indicate statistical significant differences. $* \mathrm{P}<0.05$ compared to all data points of line graph.

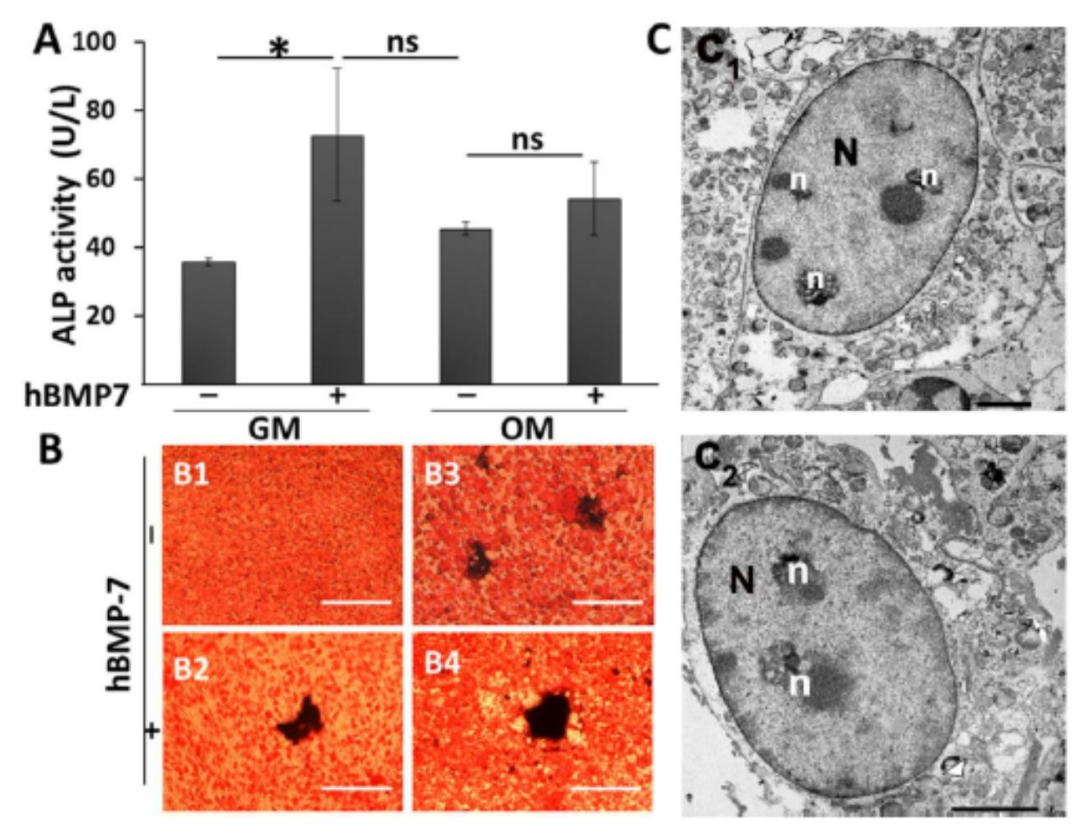


Figure 7: Osteogenic differentiation in vitro (A) Validation of ALP activity of the conditioned media of D1-MSCs, $\mathrm{n}=5$. * indicated statistical significant differences $(\mathrm{P}<$ 0.05). ns indicated no statistical significant differences ( $\mathrm{P} \geq 0.05)$. (B) Representative light micrographs of D1-MSCs culture after Von Kossa staining for calcium deposition. Scale bars $=40 \mu \mathrm{m}$. GM= Growth Medium, $\mathrm{OM}=$ Osteogenic Medium. (C) TEM micrographs for cell ultrastructure in both untransfected (C1) and transfected (C2) cultures. Arrow head points at Golgi complex, arrows point at extracellular matrix deposits. $\mathrm{N}=$ nucleus, $\mathrm{n}=$ nucleolus. Scale bars $=2 \mu \mathrm{m}$.

\section{References}

1. Attia N, Santos E, Abdelmouty H, Arafa S, Zohdy N, Hernández RM, et al. Behaviour and ultrastructure of human bone marrow-derived mesenchymal stem cells immobilised in alginate-poly-llysine- alginate microcapsules. Journal of microencapsulation. 2014;31(6):57989.

2. Vadalà G, Sowa G, Hubert M, Gilbertson LG, Denaro V, Kang JD. Mesenchymal stem cells injection in degenerated intervertebral disc: cell leakage may induce osteophyte formation. Journal of tissue engineering and regenerative medicine. 2012;6(5):348-55.

3. Yang X, Zhu T-Y, Wen L-C, Cao Y-P, Liu C, Cui Y-P, et al. Intraarticular Injection of Allogenic M esenchymal Stem Cells has a Protective Role for the Osteoarthritis. Chinese medical journal. 2015;128(18):2516.

4. Hodgkinson CP, Gomez JA, M irotsou M, Dzau VJ. Genetic engineering of mesenchymal stem cells and its application in human disease therapy. Human gene therapy. 2010;21(11):1513-26.

5. Saeed H, Ahsan M, Saleem Z, Iqtedar M, Islam M, Danish Z, et al. M esenchymal stem cells (MSCs) as skeletal therapeutics-an update. Journal of biomedical science. 2016;23(1):41.

6. Fischer J, Kolk A, Pautke C, Warnke P, Plank C, Smeets R. Future of local bone regenerationprotein versus gene therapy. Journal of Cranio-M axillofacial Surgery. 2011;39(1):54-64.

7. Zhang Y, Fan W, Ma Z, Wu C, Fang W, Liu G, et al. The effects of pore architecture in silk fibroin scaffolds on the growth and differentiation of mesenchymal stem cells expressing BM P7. Acta biomaterialia. 2010;6(8):3021-8.

8. Mont MA, Ragland PS, Biggins B, Friedlaender G, Patel T, Cook S, et al. Use of bone morphogenetic proteins for musculoskeletal applications. J Bone Joint Surg Am. 2004;86(suppl 2):41-55.

9. Kanakaris NK, Petsatodis G, Tagil M, Giannoudis PV. Is there a role for bone morphogenetic proteins in osteoporotic fractures? Injury. 2009;40:S21-S6.

10. Xiang S, Tong $H$, Shi Q, Fernandes JC, Jin T, Dai K, et al. Uptake mechanisms of non-viral gene delivery. J Control Release. 2012 Mar 28;158(3):371-8.

11. Ramamoorth $M$, Narvekar A. Non viral vectors in gene therapy-an overview. J Clin Diagn Res. 2015;9(1):GE01-GE6.

12. Oliveira AV, Marcelo A, da Costa AMR, Silva GA. Evaluation of cystamine-modified hyaluronic acid/chitosan polyplex as retinal gene vector. $M$ aterials Science and Engineering: $C$. 2016;58:264-72.

13. M arianecci C, Di Marzio L, Rinaldi F, Celia C, Paolino D, Alhaique F, et al. Niosomes from 80s to present: the state of the art. Adv Colloid Interface Sci. 2014 M ar;205:187-206.

14. Rajera R, Nagpal K, Singh SK, Mishra DN. Niosomes: a controlled and novel drug delivery system. Biol Pharm Bull. 2011;34(7):945-53.

15. Kazi KM, Mandal AS, Biswas N, Guha A, Chatterjee S, Behera M, et al. Niosome: A future of targeted drug delivery systems. J Adv Pharm Technol Res. 2010 Oct;1(4):374-80.

16. Ochoa GP, Sesma JZ, Díez MA, Díaz-Tahoces A, Avilés-Trigeros M, Grijalvo S, et al. A novel formulation based on 2, 3-di (tetradecyloxy) propan-1-amine cationic lipid combined with 
polysorbate 80 for efficient gene delivery to the retina. Pharmaceutical research. 2014;31(7):1665-75.

17. Kokotos G, Verger R, Chiou A. Synthesis of 2-Oxo Amide Triacylglycerol Analogues and Study of Their Inhibition Effect on Pancreatic and Gastric Lipases. Chemistry-A European Journal. 2000;6(22):4211-7.

18. Ojeda E, Agirre M, Villate-Beitia I, Mashal M, Puras G, Zarate J, et al. Elaboration and Physicochemical Characterization of Niosome-Based Nioplexes for Gene Delivery Purposes. Non-Viral Gene Delivery Vectors: Methods and Protocols. 2016:63-75.

19. Mashal M, Attia N, Puras G, Martínez-Navarrete G, Fernández E, Pedraz JL. Retinal gene delivery enhancement by lycopene incorporation into cationic niosomes based on DOTM A and polysorbate 60. Journal of Controlled Release. 2017.

20. Ojeda E, Puras G, Agirre M , Zárate J, Grijalvo S, Pons R, et al. Niosomes based on synthetic cationic lipids for gene delivery: the influence of polar head-groups on the transfection efficiency in HEK-293, ARPE-19 and MSC-D1 cells. Organic \& biomolecular chemistry. 2015;13(4):1068-81.

21. Rahman F, Al Frouh F, Bordignon B, Fraterno M, Landrier J-F, Peiretti F, et al. Ascorbic acid is a dose-dependent inhibitor of adipocyte differentiation, probably by reducing CAMP pool. Frontiers in cell and developmental biology. 2014;2:29.

22. Song YK, Liu D. Free liposomes enhance the transfection activity of DNA/lipid complexes in vivo by intravenous administration. Biochim Biophys Acta. 1998 J un 24;1372(1):141-50.

23. Balazs DA, Godbey W. Liposomes for use in gene delivery. Journal of drug delivery. 2010;2011.

24. Szunerits S, Boukherroub R. Introduction to Plasmonics: Advances and Applications: CRC Press; 2015.

25. Wasungu L, Hoekstra D. Cationic lipids, lipoplexes and intracellular delivery of genes. J Control Release. 2006 Nov 28;116(2):255-64.

26. Singh J, Michel D, Chitanda JM, Verrall RE, Badea I. Evaluation of cellular uptake and intracellular trafficking as determining factors of gene expression for amino acid-substituted gemini surfactant-based DNA nanoparticles. Journal of nanobiotechnology. 2012;10(1):7.

27. Majzoub RN, Ewert KK, Safinya CR. Cationic liposome-nucleic acid nanoparticle assemblies with applications in gene delivery and gene silencing. Phil Trans $R$ Soc $A$. 2016:374(2072):20150129.

28. Puras G, Mashal M, Zarate J, Agirre M, Ojeda E, Grijalvo S, et al. A novel cationic niosome formulation for gene delivery to the retina. J Control Release. 2014 Jan 28;174:27-36.

29. Sakurai F, Inoue R, Nishino Y, Okuda A, Matsumoto O, Taga T, et al. Effect of DNA/liposome mixing ratio on the physicochemical characteristics, cellular uptake and intracellular trafficking of plasmid DNA/cationic liposome complexes and subsequent gene expression. J Control Release. 2000 M ay 15;66(2-3):255-69.

30. Champion JA, Katare YK, M itragotri S. Particle shape: a new design parameter for microand nanoscale drug delivery carriers. Journal of Controlled Release. 2007;121(1):3-9.

31. Gratton SE, Ropp PA, Pohlhaus PD, Luft JC, Madden VJ, Napier ME, et al. The effect of particle design on cellular internalization pathways. Proceedings of the National Academy of Sciences. 2008;105(33):11613-8.

32. $M a$ B, Zhang $S$, Jiang $H$, Zhao $B$, Lv $H$. Lipoplex morphologies and their influences on transfection efficiency in gene delivery. Journal of Controlled Release. 2007;123(3):184-94.

33. Le Bihan $\mathrm{O}$, Chèvre $\mathrm{R}$, Mornet $\mathrm{S}$, Garnier $\mathrm{B}$, Pitard $\mathrm{B}$, Lambert $\mathrm{O}$. Probing the in vitro mechanism of action of cationic lipid/DNA lipoplexes at a nanometric scale. Nucleic acids research. 2011;39(4):1595- 609.

34. Ojeda E, Puras G, Agirre M , Zarate J, Grijalvo S, Eritja R, et al. The role of helper lipids in the intracellular disposition and transfection efficiency of niosome formulations for gene delivery to retinal pigment epithelial cells. International journal of pharmaceutics. 2016;503(1):115-26. 
35. Puras G, Martinez-Navarrete G, Mashal M, Zarate J, Agirre M, Ojeda E, et al. Protamine/DNA/Niosome Ternary Nonviral Vectors for Gene Delivery to the Retina: The Role of Protamine. Mol Pharm. 2015 Oct 5;12(10):3658-71.

36. Honda Y, Ding X, M ussano F, Wiberg A, Ho C-m, Nishimura I. Guiding the osteogenic fate of mouse and human mesenchymal stem cells through feedback system control. Scientific reports. 2013;3:3420.

37. Hsiong SX, Boontheekul T, Huebsch N, Mooney DJ. Cyclic arginine-glycine-aspartate peptides enhance three-dimensional stem cell osteogenic differentiation. Tissue Engineering Part A. 2008;15(2):263-72.

38. Garate A, Ciriza Js, Casado JG, Blazquez R, Pedraz JL, Orive G, et al. Assessment of the behavior of mesenchymal stem cells immobilized in biomimetic alginate microcapsules. Molecular pharmaceutics. 2015;12(11):3953-62.

39. Jensen EC. Use of fluorescent probes: their effect on cell biology and limitations. The Anatomical Record. 2012;295(12):2031-6.

40. El-Sayed A, Harashima H. Endocytosis of gene delivery vectors: from clathrin-dependent to lipid raft-mediated endocytosis. M olecular Therapy. 2013;21(6):1118-30.

41. Bareford LM, Swaan PW. Endocytic mechanisms for targeted drug delivery. Adv Drug Deliv Rev. 2007 Aug 10;59(8):748-58.

42. Kou L, Sun J, Zhai Y, He Z. The endocytosis and intracellular fate of nanomedicines: Implication for rational design. Asian Journal of Pharmaceutical Sciences. 2013;8(1):1-10.

43. Xu J, Wang NX, Wang MN, Xie HX, Cao YH, Sun LH, et al. BMP7 enhances the effect of BMSCs on extracellular matrix remodeling in a rabbit model of intervertebral disc degeneration. The FEBS journal. 2016;283(9):1689-700.

44. Hornstein BD, Roman D, Arévalo-Soliz LM, Engevik MA, Zechiedrich L. Effects of Circular DNA Length on Transfection Efficiency by Electroporation into HeLa Cells. PloS one. 2016;11(12):e0167537.

45. Yin W, Xiang P, Li Q. Investigations of the effect of DNA size in transient transfection assay using dual luciferase system. Analytical biochemistry. 2005;346(2):289-94.

46. Scarfi S. Use of bone morphogenetic proteins in mesenchymal stem cell stimulation of cartilage and bone repair. World journal of stem cells. 2016;8(1):1.

47. Dorman $L$, Tucci $M$, Benghuzzi $H$. In vitro effects of bmp-2, bmp-7, and bmp-13 on proliferation and differentation of mouse mesenchymal stem cells. Biomedical sciences instrumentation. 2011;48:81-7. 


\section{Graphical Abstract}

The current study presents a novel niosome formulation (DP80) based on 2,3di (tetradecyloxy) propan-1-amine cationic lipid (DTPA) and polysorbate 80 non-ionic tensioactive (P80). The cationic niosomes DP80 were used as vehicles to deliver pUNO1-hBMP-7 plasmid to D1-MSCs. Afterwards, it was confirmed that the delivered hBMP-7 gene was capable of enhancing cell proliferation, increasing ALP activity and inducing spontaneous osteogenic differentiation as observed by deposition of calcified extracellular matrix seen by LM (Von Kossa staining) and TEM.

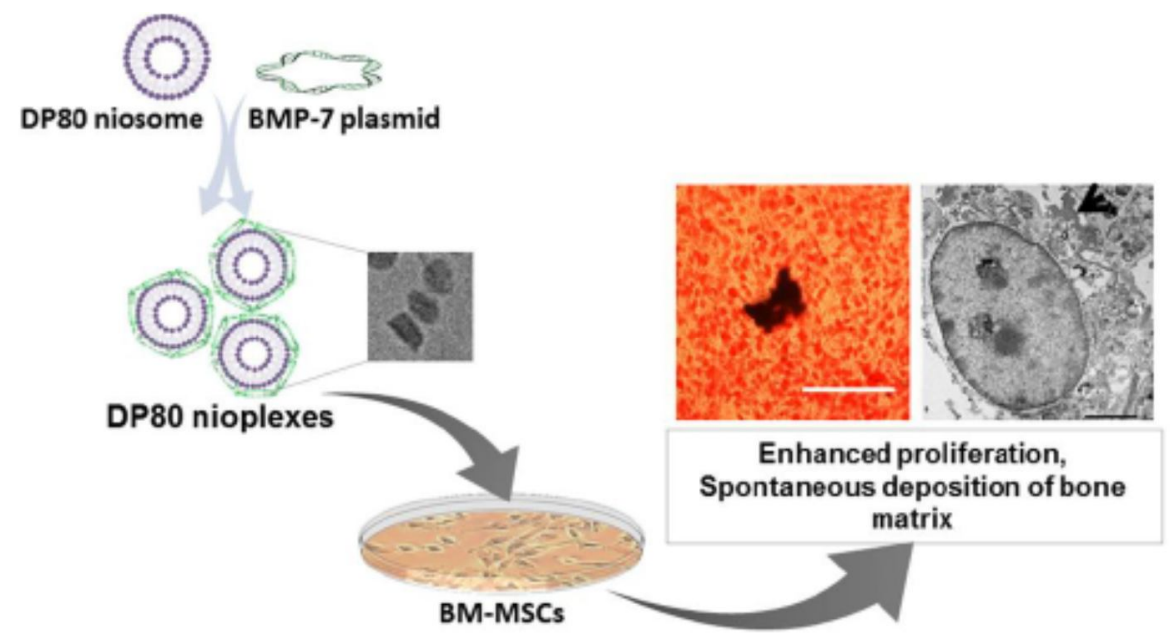

